\title{
The young star cluster system of the Antennae galaxies
}

\author{
Peter Anders • Uta Fritze • Richard de Grijs
}

Received: 25 January 2008 / Accepted: 2 April 2008 / Published online: 22 September 2009

(C) The Author(s) 2009. This article is published with open access at Springerlink.com

\begin{abstract}
The study of young star cluster (YSC) systems, preferentially in starburst and merging galaxies, has seen great interest in the recent past, as it provides important input to models of star formation. However, even some basic properties (such as the luminosity function; LF) of YSC systems are still being debated. Here, we study the photometric properties of the YSC system in the nearest major merger system, the Antennae galaxies. We find evidence for the existence of a statistically significant turnover in the LF.
\end{abstract}

Keywords Star clusters: general · Galaxies: NGC 4038/39 - Methods: data analysis

\section{Introduction}

Star clusters (SCs) form nearly instantaneously through the collapse of giant molecular gas clouds. Hence, all stars within a SC are approximately coeval, share the same chemical composition, and therefore represent a simple stellar

P. Anders $(\bowtie)$

Sterrenkundig Instituut, Universiteit Utrecht, P.O. Box 80000, 3508 TA Utrecht, The Netherlands

e-mail: P.Anders@phys.uu.nl

U. Fritze

Centre for Astrophysics Research, University of Hertfordshire, College Lane, Hatfield AL10 9AB, UK

R. de Grijs

Department of Physics \& Astronomy, The University of Sheffield,

Hicks Building, Hounsfield Road, Sheffield S3 7RH, UK

R. de Grijs

National Astronomical Observatories, Chinese Academy

of Sciences, 20A Datun Road, Chaoyang District,

Beijing 100012, China population. A small number of parameters, in particular their initial chemical composition and stellar initial mass function, are sufficient to describe their colour and luminosity evolution on the basis of a given set of stellar evolutionary tracks or isochrones (e.g. Leitherer et al. 1999; Anders and Fritze-v. Alvensleben 2003; Bruzual and Charlot 2003). Therefore, observed spectrophotometric properties of SCs are relatively easy and straightforward to interpret.

$\mathrm{SC}$ formation is a major mode of all star formation, and possibly even the dominant mode in strong starbursts triggered in gas-rich galaxy mergers (e.g. Meurer 1995; de Grijs et al. 2003b). In addition, as SCs inherit and conserve the chemical abundances at the place and time of their birth until old age, they are excellent tracers of their parent galaxy's properties in terms of the star formation and chemical enrichment history.

One of the most basic and commonly used diagnostics to explore the properties of entire SC systems is their luminosity function (LF). While for old globular cluster systems the Gaussian shape of their LFs (and, indeed, of the mass functions; MFs) is well established (see, e.g. Ashman and Zepf 1998; Harris 1991), the situation for young SCs (YSCs) is still vigorously being discussed. Whereas mainly LFs consistent with a power-law, resembling the MF of nearby molecular cloud cores, are quoted for YSC systems ranging from Galactic open cluster to YSCs in major mergers (e.g. van den Bergh and Lafontaine 1984; Hunter et al. 2003; Schweizer and Seitzer 1998; Whitmore et al. 1999; see de Grijs et al. 2003a for a recent compilation), some studies find deviations from a power-law, or even direct evidence for Gaussian distributions (de Grijs and Anders 2006; Fritze-v. Alvensleben 1999; Goudfrooij et al. 2004).

To contribute to our understanding of this astrophysical question, which directly ties in to the fundamental physical conditions of star formation in general, we performed 
an analysis of the young ( $\sim-100 \mathrm{Myr}) \mathrm{SC}$ system formed during the - still ongoing-merging process in the nearest major merger of two giant gas-rich spiral galaxies, the Antennae system, NGC4038/39.

\section{Photometry}

We reanalysed the most homogeneous broad-band data set including the $U$-band of the Antennae galaxies available (providing photometry in $U B V I$ ), obtained using the $H u b$ ble Space Telescope's Wide-Field and Planetary Camera-2 (WFPC2) as part of programme GO-5962 (PI B. Whitmore).

We selected our clusters based on:

- A flux threshold compared to its surrounding

- Cross-correlation between filters (i.e., a cluster must be detected in several pass bands)

- Photometric errors $<0.2$ mag in each filter; and

- Size measurements using BAOLAB (Larsen 1999)

Selecting only clusters in the (PSF-corrected) size range $R_{1 / 2} \simeq 5$ to $25 \mathrm{pc}$ (to avoid contamination by single stars and cluster complexes, respectively) yields a sample of 365 clusters, satisfying all selection criteria. Utilising the cluster sizes we applied size-dependent aperture corrections, as described in Anders et al. (2006).

\section{Completeness}

In this study we are primarily interested in the LF of the YSCs. A consistent and reliable determination of the completeness fraction as a function of cluster magnitude and all other relevant selection criteria and effects is therefore of prime importance.

We investigated the completeness for a number of restrictions, taking successively more cluster selection criteria into account. Some of these results, for artificial clusters with $\mathrm{FWHM} \simeq 10 \mathrm{pc}$ in a cluster-rich region in NGC4038, are shown in Fig. 1.

First, we determined the completeness in each band independently (labelled 'U', 'B', 'V' and 'I' in Fig. 1). Taking the cross-correlation into account (labelled 'XID') reduced the completeness to slightly below the most limiting singleband completeness. By applying the photometric uncertainties restriction (labelled 'XID + photo') the 50\% completeness magnitude decreases by about $\simeq 0.4 \mathrm{mag}$.

Using stars instead of extended objects as test sources artificially increases the $50 \%$ completeness magnitude by $\simeq 1 \mathrm{mag}$.

Computing completeness functions for two regions (characterised by different background and cluster density levels) and two sizes (characteristic for our observed cluster

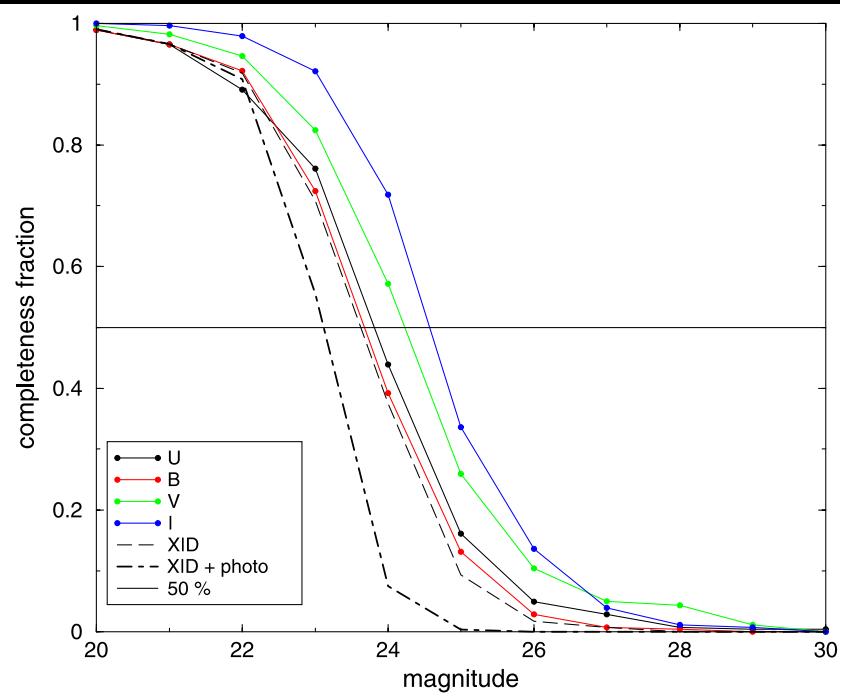

Fig. 1 Completeness fractions for artificial clusters with $\mathrm{FWHM} \simeq 10 \mathrm{pc}$ for a variety of selection criteria (see text for details)

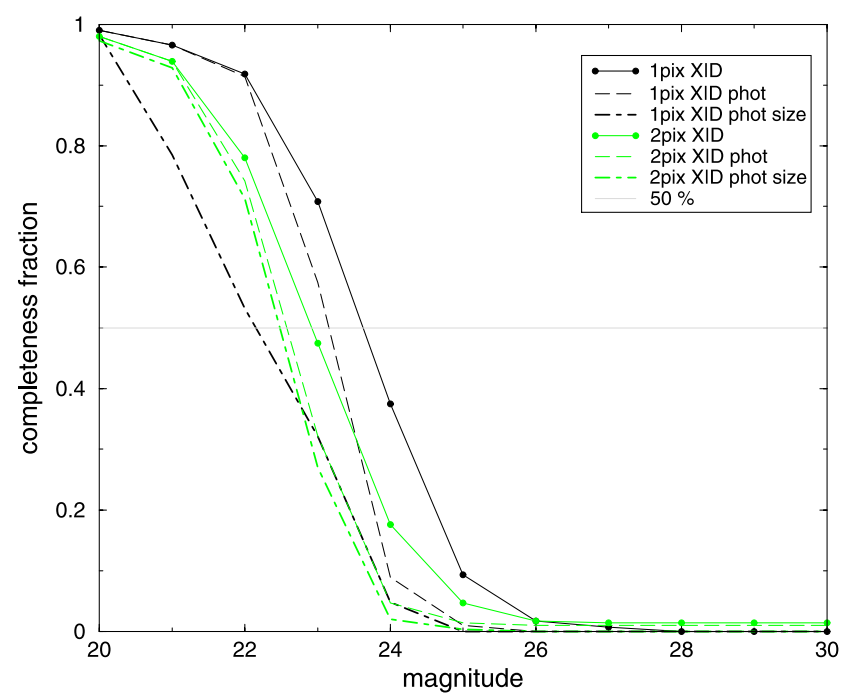

Fig. 2 Completeness fractions for artificial clusters with $\mathrm{FWHM} \simeq 10 \mathrm{pc}(=1$ pixel $)$ and $\mathrm{FWHM} \simeq 20 \mathrm{pc}(=2$ pixels $)$ for a variety of selection criteria (see text for details)

sample), and imposing the size restriction onto the artificial clusters (labelled 'XID photo size') allowed us to attribute realistic completeness fractions to each individual cluster, strengthening the subsequent analysis of the LFs. Imposing the size restrictions decreases the completeness by up to $\simeq 1 \mathrm{mag}$, while different cluster sizes and regions within the galaxies contribute completeness changes of $\simeq 0.6$ and $\simeq 1 \mathrm{mag}$, respectively. This very complex dependence of the completeness fractions on a number of input parameters clearly shows the necessity to model the completeness functions as realistically as possible, taking into account all cluster selection criteria. For the same region as in Fig. 1, results for different cluster sizes and selection criteria are shown in Fig. 2. 
For additional details of the completeness determination, see Anders et al. (2007).

\section{The model to analyse the luminosity functions}

In collaboration with statisticians from the University of Göttingen we developed a suite of statistical tools. This allows for the fitting of the LFs of our YSCs with either a Gaussian distribution or a power law, assess the accuracy of the fits and the resulting parameters, and establish the statistical significance of differences in the goodness-of-fit between the two test distributions.

The fitting of an observed LF takes the photometric error and appropriate completeness fraction of each individual cluster into account, and independently fits either a Gaussian distribution or a power law to the LF, yielding a set of parameters characterising the best-fitting model and a likelihood parameter for either test distribution.

We find a strong superiority (as measured by the ratio of the likelihoods for Gaussian and power-law fits) of the Gaussian compared to the power-law fit (see Sect. 5). To validate this result, we have to test its statistical significance, i.e., we have to determine the probability of achieving such a strong superiority of the Gaussian distribution if the true underlying LF is a power law, distorted by the error distribution and the completeness function. In a Monte Carlo approach we draw artificial observations from the best-fitting power law, use our statistics tools to fit them again with Gaussian and power-law distribution, and determine the likelihood ratio for these artificial observations. The likelihood ratio of the observed distribution compared to the distribution of likelihood ratios from our artificial tests then yields the probability that the observed superiority of the Gaussian fits is consistent with an underlying power-law distribution.

The uncertainties of the best-fitting model parameters are estimated by bootstrapping. For additional details on the statistical model, see Anders et al. (2007).

\section{Results}

As an example, the $V$-band LF for our YSC sample in the Antennae galaxies is shown in Fig. 3. From visual inspection, the best fit assuming an underlying Gaussian distribution appears to represent the data better than the best fit based on an underlying power-law distribution.

To quantify this, we perform our likelihood-ratio test and the Monte Carlo analysis. For the observed distributions we find a likelihood-ratio value of 23.1 (where larger values are equivalent to a stronger superiority of the Gaussian compared to the power-law fit). Our Monte Carlo analysis with 1000 test realisations drawn from the best-fitting power-law
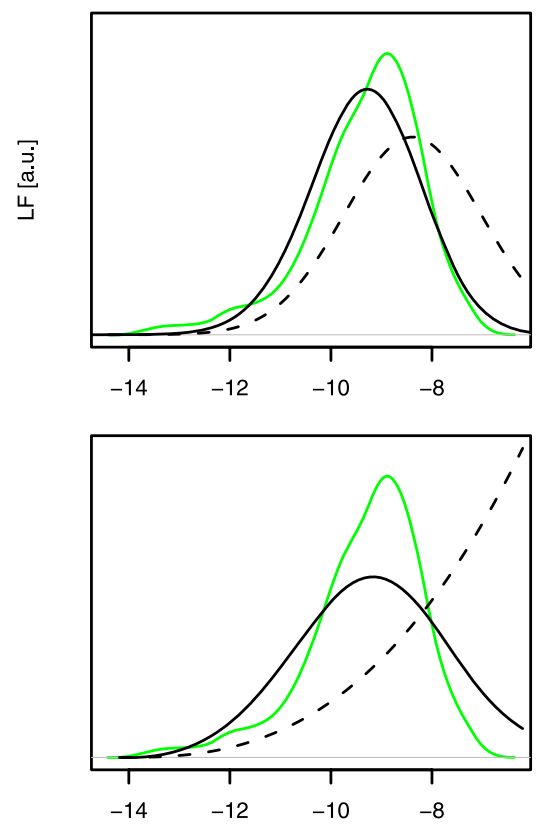

Fig. $3 V$-band luminosity functions for our Antennae clusters and fit: observed (green solid line), best-fit (black solid) and underlying distribution of best fit (black dashed). Fitted test distributions are the Gaussian (left panel) and power-law (right panel) realisations

distribution results in a maximum likelihood ratio of 11.4; hence none of the test distributions can reproduce a superiority of the Gaussian distribution as strong as observed. Utilising the properties of Bernoulli-distributed variables, this corresponds to a probability of $<0.5 \%$ that the underlying distribution is still consistent with a power law.

This result is also valid for the other bands (except for the $I$-band, for which the observations are significantly shallower), and for several age and size subsamples. For more details, see Anders et al. (2007).

Acknowledgements We would like to thank Nicolai Bissantz and Leif Boysen for their constant help and for the development of the statistical tools.

Open Access This article is distributed under the terms of the Creative Commons Attribution Noncommercial License which permits any noncommercial use, distribution, and reproduction in any medium, provided the original author(s) and source are credited.

\section{Appendix: Discussion}

Fritze: Do you have any ideas as to how to eventually translate the completeness limit in luminosity into one in mass?

Anders: Yes, but only ideas so far. One would need to assign an individual completeness fraction to every cluster, based on its luminosity. This completeness fraction would need to 
be retained throughout the entire analysis of the cluster, including the luminosity-to-mass conversion. However, in this case we cannot talk about a completeness function anymore.

Whitmore: I liked the demonstration of how messy the completeness correction is, depending on background, cluster size, etc. It can vary by several magnitudes, and hence simply representing it by a single line (as we often do) is not the whole story. A comment on your approach to differentiating between a power law and a Gaussian: at the bright end, they both have roughly the same shape, so it is really just the small $\sim 1$ mag range where completeness comes in where all the diagnostic power is. Therefore, it comes down to trying to compare very small differences over a small range to tell the difference between power law and Gaussian. And as we just mentioned, getting the completeness correction exactly right is very difficult. A more robust approach is to push the distribution a few magnitudes deeper, which we have now done using our ACS data, demonstrating that the distribution continues to be a power law a few magnitudes beyond where your implied cut-off is.

Anders: I agree that completeness determination and subsequent Gaussian or power-law fitting is a tricky business. As your deep ACS data were not available when I started this project, I had to deal with the older WFPC2 data. If the new data disprove my results from older observations, then I accept that as scientific progress. I am still concerned about stellar contamination, though.

\section{References}

Anders, P., Fritze-v. Alvensleben, U.: Astron. Astrophys. 401, 1063 (2003)

Anders, P., Gieles, M., de Grijs, R.: Astron. Astrophys. 451, 375 (2006)

Anders, P., Bissantz, N., Boysen, L., de Grijs, R., Fritze-v. Alvensleben, U.: Mon. Not. R. Astron. Soc. 377, 91 (2007)

Ashman, K.M., Zepf, S.E.: Globular Cluster Systems. Cambridge Astrophysics Series, vol. 30. Cambridge University Press, Cambridge (1998)

Bruzual, G., Charlot, S.: Mon. Not. R. Astron. Soc. 344, 1000 (2003)

de Grijs, R., Anders, P.: Mon. Not. R. Astron. Soc. 366, 295 (2006)

de Grijs, R., Anders, P., Bastian, N., Lynds, R., Lamers, H.J.G.L.M., O’Neil, E.J.: Mon. Not. R. Astron. Soc. 343, 1285 (2003a)

de Grijs, R., Lee, J.T., Clemencia Mora Herrera, M., Fritze-v. Alvensleben, U., Anders, P.: New Astron. 8, 155 (2003b)

Fritze-v. Alvensleben, U.: Astron. Astrophys. 342, L25 (1999)

Goudfrooij, P., Gilmore, D., Whitmore, B.C., Schweizer, F.: Astrophys. J. Lett. 613, L121 (2004)

Harris, W.E.: Annu. Rev. Astron. Astrophys. 29, 543 (1991)

Hunter, D.A., Elmegreen, B.G., Dupuy, T.J., Mortonson, M.: Astron. J. 126, 1836 (2003)

Larsen, S.S.: Astron. Astrophys. Suppl. Ser. 139, 393 (1999)

Leitherer, C., Schaerer, D., Goldader, J.D., Delgado, R.M.G., Robert, C., Kune, D.F., de Mello, D.F., Devost, D., Heckman, T.M.: Astrophys. J. Suppl. Ser. 123, 3 (1999)

Meurer, G.R.: Nature 375, 742 (1995)

Schweizer, F., Seitzer, P.: Astron. J. 116, 2206 (1998)

van den Bergh, S., Lafontaine, A.: Astron. J. 89, 1822 (1984)

Whitmore, B.C., Zhang, Q., Leitherer, C., Fall, S.M., Schweizer, F., Miller, B.W.: Astron. J. 118, 1551 (1999) 